\title{
ALL NETS ARE EQUAL, BUT SOME NETS ARE MORE EQUAL THAN OTHERS
}

\author{
Mark Rowland Editorial Board Member, LSHTM
}

Keywords: malaria, mosquito, vector control, insecticide treated net, PBO, piperonyl butoxide

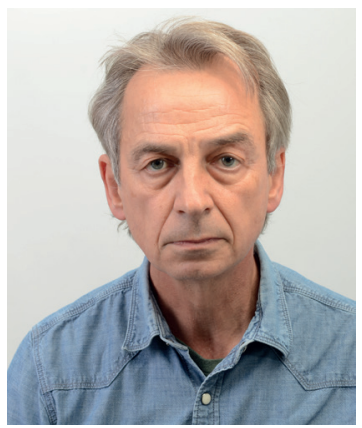

Mark Rowland

It is almost a cliché to open any popular article on malaria vector control by attributing much of the dramatic decline in malaria in sub-Saharan Africa since 2000 to the massive investment in long-lasting insecticide treated nets (LLIN) (Bhatt et al. 2015). According to the latest figures from the World Health Organization (WHO), over half of Africa's population now has access to LLIN, increasing from $33 \%$ in 2010 to $57 \%$ in 2019 (WHO 2019). In 2018 alone, 197 million LLINs were delivered to Africa by manufacturers. Despite this, LLIN coverage has improved only marginally since 2015. The malaria burden worldwide has fallen only slightly from an estimated 231 million cases of malaria in 2017 to 228 million in 2018, and is at a standstill in Africa. Resistance to pyrethroids - the only insecticide class currently used routinely in LLIN - is widespread and continues to increase in frequency in many countries. While resistance to pyrethroids acts as a break on progress it is not the only factor impeding progress. The physical durability of the nets (2-3 years before nets become holed and unserviceable) is proving shorter than the ability of many countries to implement net distribution campaigns to the population at risk (3-5 years). Holed nets are less protective against mosquitoes that are strongly pyrethroid resistant (N'Guessan et al. 2007). Holed nets and net shortages may lead to periods of inadequate personal and community protection, and to temporary increases in malaria morbidity between distribution campaigns.

In an editorial a year ago, we discussed the findings of a cluster-randomised trial of a new type of ITN in which the synergist PBO (piperonyl butoxide) and pyrethroid (permethrin) were incorporated into the net fibre to overcome resistance to pyrethroids mediated by mono-oxygenase enzymes (Outlooks on Pest Management 2018). Randomised trials are regarded as the gold standard for assessing evidence of efficacy and for gener-

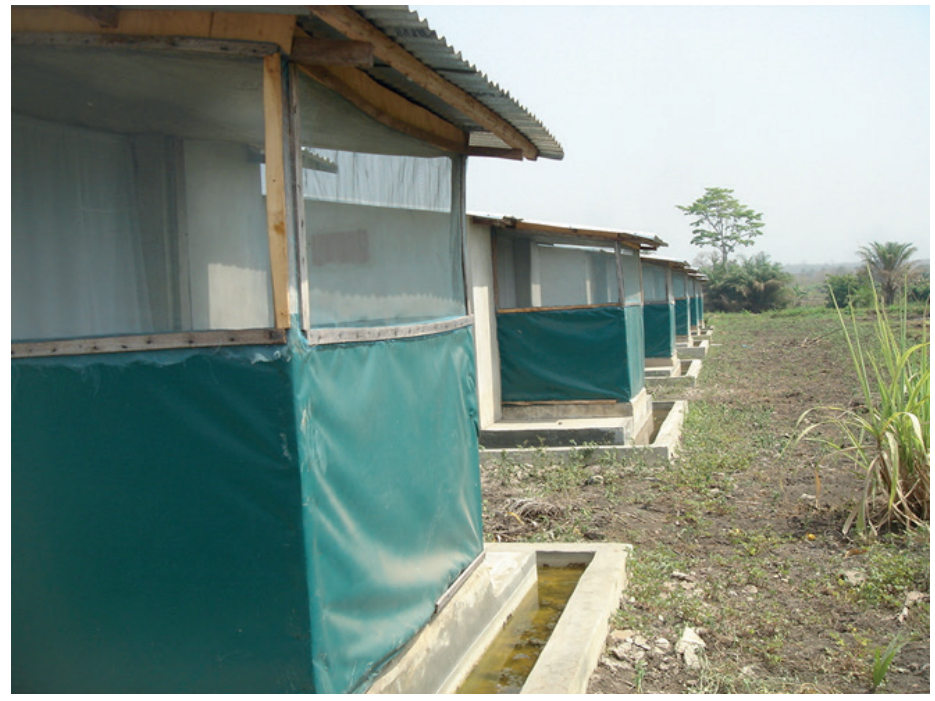

Suite of experimental huts and veranda traps for testing mosquito nets and collecting mosquitoes in West Africa.

ating unbiased estimates of effect sizes of new vector control tools. Since 2017, WHO has made randomised trial evidence a policy requirement before recommending any new class of net (WHO 2017). In the Tanzanian trial reported earlier, the villages that were randomised to receive the pyrethroid-PBO synergist net recorded a prevalence of malaria infection after 9 months of use $44 \%$ less than the villages randomised to the standard pyrethroid LLIN (prevalence ratio $0.56,95 \%$ confidence interval 0.40-0.77) (Protopopoff et al. 2018). After 21 months of use, the prevalence was still $33 \%$ less (PR 0.67, CI 0.50-0.90). On the basis of these results, WHO awarded interim 'new vector control product class' status to pyrethroidPBO ITN. In response to the WHO recommendation, the main international malaria control agencies - the Global Fund and the US Present's Malaria Initiative (PMI) - started to place orders for this new class of net.

Before giving recommendation to a new product class WHO normally expects randomised trial evidence from two studies from different places. While the WHO made an exception owing to the pressing need to introduce a new class of active net, a second randomised trial of pyrethroid-PBO ITN has recently reported results from Uganda (Staedke et al. $\left.2019 a^{1}\right)$. What did it show? This trial was innovative for being embedded within the national LLIN distribution campaign across over a hundred clusters (health sub-districts) of the East and West Regions of Uganda (Staedke et al. 2019b). It involved two types of PBO-pyrethroid ITN: Olyset Plus and PermaNet 3, and two types of standard pyrethroid only LLIN:

1. This is preliminary research that has not been peer-reviewed. 
Olyset Net and PermaNet 2. Olyset Plus and Olyset Net were the same nets trialled earlier in Tanzania. PermaNet 3 differs from Olyset Plus in restricting the PBO synergist to the roof panel which mosquitoes contact in response to plumes of human odour from inside the net. Side panels of PermaNet 3 are treated with pyrethroid alone (deltamethrin) at a slightly higher concentration than in the standard LLIN PermaNet 2. PermaNet 3 and PermaNet 2 formed twice as many clusters as Olyset Plus and Olyset Net in the 4 arm trial.

Surveys of malaria parasite infection in children were undertaken 6,12 and 18 months after LLIN distribution to the communities. Overall, the prevalence of malaria infection in surveys at 6,12 and 18 months was lower in the PBO arm than in the non-PBO arm. In the sub-group analysis stratified by manufacturer, the prevalence of malaria infection was lower in PermaNet 3 (with PBO) at 6 and 12 months than in PermaNet 2 but no difference in effect was evident at 18 months. For the Olyset arms, malaria infection prevalence was lower in Olyset Plus (with PBO) after 12 and 18 months of use compared to Olyset Net, and this trend was consistent with that previously reported in the Tanzanian trial of Olyset Plus vs Olyset Net after surveys at 9 and 21 months. The non-significant difference between the Olyset Plus and Olyset Net arms in the initial post-intervention survey at 6 months in Uganda was consistent with the initial survey at 4 months in Tanzania.

Why is PermaNet 3 effective at 6 and 12 months and appears less effective at 18 ? Why is Olyset Plus effective at 12 months, still effective at 18 months but seemingly less effective at 6-months? Despite some imbalances in the sizes of arms and regions which the authors state prevents drawing conclusions between brands, one reason may lie less in the epidemiology but more in the initial laboratory assessments of insecticide/PBO durability and retention that was commissioned by the WHO Pesticide Evaluation Scheme on PermaNet 3 and Olyset Plus in their initial evaluations a decade ago (WHO 2009, 2012). To assess the durability and wash resistance of active ingredients in a new brand of LLIN, the standard WHO practice is to subject samples of netting to a series of 20 standardised soap washings and determine every 3-5 washes the residual insecticide and synergist remaining in the netting using chemical analysis and mosquito bioassay. The producers of LLINs are careful to balance the loading dose and migration of the active ingredients from the 'reservoir' within the fibre to the surface where it is bioavailable to mosquito contact. Migration too fast and reservoir of AI is depleted before the nets' physical lifespan is reached. Migration too slow and the AI is locked in the fibre reservoir and biologically unavailable to the surface. The results showed that in the Olyset Plus netting the piperonyl butoxide retention after 20 washes was $44.2 \%$, corresponding to an average retention per wash of $96.0 \%$, and continued to migrate with further washing. But in the PermaNet 3 roof-netting the piperonyl butoxide retention after 20 washes was $76.0 \%$, corresponding to a much higher retention per wash of $98.6 \%$, and according to the WHO Working Group report 'after 15 washes no further release of $\mathrm{PBO}$ seemed to occur'. Retention of pyrethroid at 20 washes was consistent with that of $\mathrm{PBO}$, i.e. $64.1 \%$ retention of pyrethroid permethrin in Olyset Plus and $89.5 \%$ retention of pyrethroid deltamethrin in PermaNet 3. These trends indicated to the WHO Working Group that

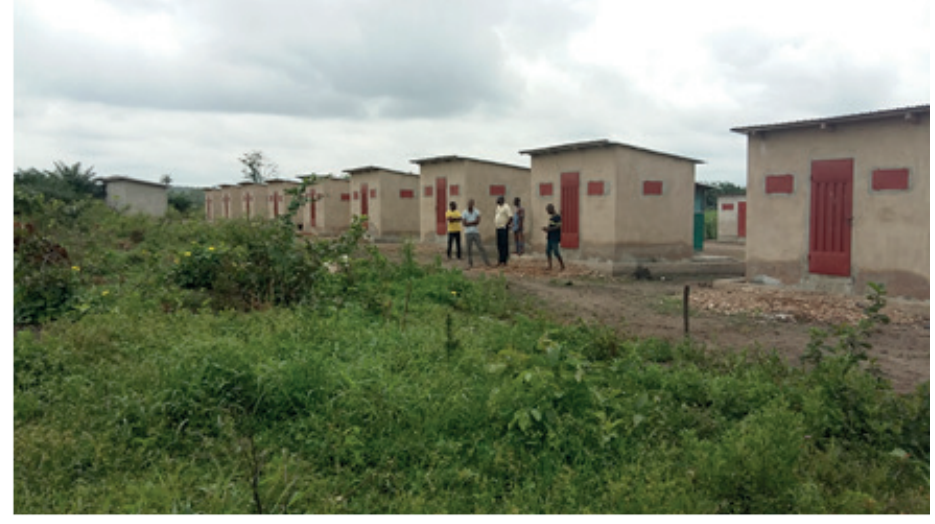

Suite of experimental huts for testing mosquito nets, West Africa.

the migration of active ingredients to the surface of fibre appeared less bound in Olyset Plus than in PermaNet 3 over the course of 20 washes.

The chemical analysis is direct evidence of AI retention and indirect evidence of release to the surface of the fibre. The next stage in the WHO assessment of new types of LLINs (Phase 2) are experimental hut trials. These are entomological trials done under controlled household conditions in special huts in which human volunteers sleep under the study nets and free-flying wild mosquitoes are attracted into the huts to feed each night. PBOpyrethroid LLIN are compared against standard pyrethroid LLIN when new and unwashed and after 20 wash cycles. Parallel trials are undertaken across a range of countries to build the evidence. What did the field washing and household efficacy trials show? Firstly, that field washes gave retention rates consistent with the laboratory washing: $\mathrm{PBO}$ retention was lower in Olyset Plus at $45 \%$ and higher in PermaNet 3 at $78 \%$. Similarly, pyrethroid retention was lower at $76 \%$ in Olyset Plus and higher at $85 \%$ in PermaNet 3 . Two efficacy outcomes were assessed in the huts: mosquito mortality rate and mosquito blood feeding rate through the nets. A Cochrane meta-analysis summarised the results (Gleave et al. 2018). The comparison of $\mathrm{PBO}$ nets versus non-PBO nets is presented as summary risk ratios of mosquito mortality and blood feeding. Mortality risk ratios significantly greater than 1.0 indicate greater control of mosquitoes by PBO nets, blood-feeding risk ratios significantly less than 1.0 indicate greater personal protection from biting by $\mathrm{PBO}$ nets. Comparing Olyset Plus versus Olyset Net the mortality rate ratio was significantly greater than 1.0 both before (1.72, CI 1.48-2.0) and after (1.81, CI 1.25-2.61) the series of standardized washing indicating continuing presence of surface PBO on Olyset Plus. Comparing PermaNet 3 versus PermaNet 2 the mortality risk ratio was significantly greater before (1.81, CI 1.56-2.10) but non-significant $(1.18$, CI 0.612.28) after the washing indicating loss of surface PBO. For the blood-feeding risk ratios between Olyset Plus versus Olyset Net the ratio was 0.67 (CI $0.38-1.18$ ) before and 0.50 (CI 0.270.93 ) after washing, and between PermaNet 3 and 2 the risk ratio was 0.53 (CI $0.40-0.69$ before and 0.76 (CI 0.61-0.93) after. Both types of Pyrethroid-PBO LLIN were therefore still protective against blood-feeding after washing compared to the standard LLIN counterparts.

The problem flagged up by the review is that few studies progressed to comparing washed nets in experimental huts which is important to showing $\mathrm{PBO}$ migration and retention 
at the net surface; only one of the Olyset trials and two of the PermaNet trials compared this in high resistance area. No metaanalysis is definitive or conclusive after so few studies. Also none of these experimental hut studies on Olyset Plus and PermaNet 3 before and after washing was directly comparative at the same location at the same time or yielded a complete dataset. It was surprising that southern Benin was classified as mid resistance in the review (Gleave et al. 2018) when it was the first country in West Africa to show operational survival of vector mosquitoes due to pyrethroid resistance in experimental huts as early as 2007 (N'Guessan et al. 2007; 2010). Had the division between mid and high resistance been made on the basis of observed mortality response in huts to pyrethroid-only nets the division between high and mid would have merged giving more power.

The outcome of the Ugandan trial may confirm WHO recommendation for pyrethroid-PBO LLIN as a new class of LLIN and Olyset Plus as the first-in-class following further review. A feature common to the two cluster randomised trials in Uganda and Tanzania that remains curious is the lag between the distribution of the Olyset Plus and the effect on malaria prevalence. The effect is not evident by the time of first survey at 6 months in Uganda. PermaNet 3 had a more immediate impact, by the $6^{\text {th }}$ month survey. In Tanzania a trend was observed at the 4th month survey but it was not significant until the $2^{\text {nd }}$ survey done in the $9^{\text {th }}$ month (Protopopoff et al. 2018).

WHO Vector Control-Prequalification Team has five brands of Pyrethroid-PBO ITNs presently listed for use. These include Olyset Plus and PermaNet 3 and three other brands made by other manufacturers. In all, there are three pyrethroids in use (permethrin, deltamethrin, alpha-cypermethrin), two modes of PBO presentation (roof panel only, all 5 panels), six dosages of deltamethrin and five dosages of PBO. Decisions have to be taken. WHO is not expecting each of these brands to undergo epidemiological evaluation versus the first-in-class. Time and development costs would be prohibitive. Instead, WHO policy is to assess candidate 2nd-in-class products for entomological efficacy only (WHO 2018). Due to the significant variation in the specifications of the candidates, to generate the required assurance of comparative performance to $1^{\text {st }}$ in class, WHO has designed a noninferiority trial design to demonstrate whether each candidate $2^{\text {nd }}$-in-class test product is no worse in experimental hut trials than the $1^{\text {st }}$-in-class product by more than a specified noninferiority margin defined as an odds ratio of 0.7 between the $1^{\text {st }}$ - and $2^{\text {nd }}$-in-class interventions. This value is the maximum odds ratio that is considered acceptable for two products to be in the same policy class, and is a compromise between the risk of accepting an inferior product and the feasibility of conducting the trials. To be defined as non-inferior, a new product must show non-inferiority in terms of both mosquito mortality and blood-feeding rate. Two series of experimental hut trials of the 5 brands of Pyrethroid-PBO ITN both in unwashed state and after 20 standardised washes are due to start shortly in Côte d'Ivoire and Tanzania in W and E Africa. Because there is a question mark hanging over the veracity of 20 standardised washes as a true surrogate of wear-andtear under household conditions, it is intended to also supply the 5 brands to neighbouring households at the experimental hut sites and re-test in the huts after one year of use to verify whether they are non-inferior to the 20-times washed nets.

\section{References}

Bhatt, S., Weiss, D.J., Cameron, E., et al. (2015) The effect of malaria control on Plasmodium falciparum in Africa between 2000 and 2015. Nature, 526(7572): 207-211.

Gleave, K., Lissenden, N., Richardson, M., Choi, L., Ranson, H. (2018) Piperonyl butoxide (PBO) combined with pyrethroids in insecticide-treated nets to prevent malaria in Africa. Cochrane Database Syst Rev 11: CD012776.

N’Guessan, R., Corbel, V., Akogbéto, M., Rowland., M. (2007) Reduced Efficacy of Insecticide Treated Nets and Indoor Residual Spraying for Malaria Control in Pyrethroid Resistance Area, Benin. Emerging Infectious Diseases, 13, 199-206.

N'Guessan, R., Asidi, A., Boko, P., Odjo, A., Akogbeto, M., Pigeon, O., et al. (2010) An experimental hut evaluation of PermaNet 3.0, a deltamethrin-piperonyl butoxide combination net, against pyrethroid-resistant Anopheles gambiae and Culex quinquefasciatus mosquitoes in southern Benin. Trans. Roy. Soc. Trop. Med. Hyg.; 104(12): 758-765.

Protopopoff, N., Mosha, J.F., Lukole, E., Charlwood, J.D., Wright, A., Mwalimu, C.D., Manjurano, A., Mosha, F.W., Kisinza, W., Kleinschmidt, I., Rowland, M. (2018) Effectiveness of a longlasting piperonyl butoxide-treated insecticidal net and indoor residual spray interventions, separately and together, against malaria transmitted by pyrethroid-resistant mosquitoes: a cluster, randomised controlled, two-by-two factorial design trial. Lancet; 391: 1577-88.

Rowland., M., Protopopoff, N., (2018). Dawn of the PBO-Pyrethroid Long Lasting Net - Light at Last. Outlooks on Pest Management, 29(6), 242-244.

Staedke, S., Gonahasa, S., Dorsey, G., Kamya, M.R., Maiteki Sebuguz, C., Lynd, A., Katureebe, A., Kyohere, M., Mutungi, P., Kigozi, S.P., Opigo, J., Hemingway, J., Donnelly, M.J. (2019) LLIN Evaluation in Uganda Project (LLINEUP) - Effect of Long-Lasting Insecticidal Nets (LLINs) with, and Without, Piperonyl Butoxide on Malaria Indicators in Uganda: A ClusterRandomised Trial Embedded in a National LLIN Distribution Campaign (November 22, 2019). Available at SSRN: https:// ssrn.com/abstract $=3491911$. This is preliminary research that has not been peer-reviewed.

Staedke, S.G., Kamya, M.R., Dorsey, G., et al. LLIN (2019). Evaluation in Uganda Project (LLINEUP) - Impact of longlasting insecticidal nets with, and without, piperonyl butoxide on malaria indicators in Uganda: study protocol for a clusterrandomised trial. Trials 20(1): 321.

WHO (2009) Report of the Twelfth WHOPES Working Group Meeting, 8-11 December 2008, Review of: Bioflash GR, Permanet 2.0, Permanet 3.0, Permanet 2.5, Lambda-cyhalothrin LN. WHO/HTM/NTD/WHOPES/2012.5. Geneva, Switzerland: World Health Organization.

WHO (2012) Report of the Fifteenth WHOPES Working Group Meeting WHO/HQ, Geneva, 18-22 June 2012. Review of: Olyset PLUS, Interceptor LN, Malathion 440 EW, Vectobac GR. WHO/HTM/NTD/WHOPES/2009.1. Geneva, Switzerland: World Health Organization.

WHO (2017) Design of epidemiological trials for vector control products, Report of a WHO Expert Advisory Group. Geneva, Switzerland: World Health Organization.

WHO (2018) Data requirements and protocol for determining non-inferiority of insecticide-treated net and indoor residual spraying products within an established WHO policy class. Geneva, Switzerland: World Health Organization.

WHO (2019) World malaria report 2019. Geneva, Switzerland: World Health Organization. 